\title{
Increased immunoreactivity of c-Fos in the spinal cord of the aged mouse and dog
}

\author{
JI HYEON AHN ${ }^{1 *}$, MYOUNG CHUL SHIN ${ }^{2 *}$, JOON HA PARK ${ }^{1}$, IN HYE KIM ${ }^{1}$, JAE-CHUL LEE ${ }^{1}$, \\ BING CHUN YAN ${ }^{3}$, IN KOO HWANG ${ }^{4}$, SEUNG MYUNG MOON ${ }^{5}$, JI YUN AHN ${ }^{2,6}$, TAEK GEUN OHK ${ }^{2,7}$, \\ TAE HUN LEE ${ }^{8}$, JUN HWI CHO ${ }^{2}$, HYUNG-CHEUL SHIN ${ }^{9}$ and MOO-HO WON ${ }^{1}$
}

\begin{abstract}
Departments of ${ }^{1}$ Neurobiology, and ${ }^{2}$ Emergency Medicine, School of Medicine, Kangwon National University, Chuncheon, Gangwon 200-701, Republic of Korea; ${ }^{3}$ Institute of Integrative Traditional and Western Medicine, Medical College,

Yangzhou University, Yangzhou, Jiangsu 225001, P.R. China; ${ }^{4}$ Department of Anatomy and Cell Biology, College of Veterinary Medicine, Seoul National University, Seoul 151-742; ${ }^{5}$ Department of Neurosurgery,

Dongtan Sacred Heart Hospital, College of Medicine, Hallym University, Hwaseong, Gyeonggi 445-170; ${ }^{6}$ Department of Emergency Medicine, Sacred Heart Hospital, College of Medicine, Hallym University, Anyang, Gyeonggi 431-796; ${ }^{7}$ Department of Emergency Medicine, Kangnam Sacred Heart Hospital, College of Medicine, Hallym University, Seoul 150-950; Departments of ${ }^{8}$ Emergency Medicine, Chuncheon Sacred Heart Hospital, and

${ }^{9}$ Physiology, College of Medicine, Hallym University, Chuncheon, Gangwon 200-702, Republic of Korea
\end{abstract}

Received April 10, 2014; Accepted July 22, 2014

DOI: $10.3892 / \mathrm{mmr} .2014 .2800$

\begin{abstract}
Expression of c-Fos in the spinal cord following nociceptive stimulation is considered to be a neurotoxic biomarker. In the present study, the immunoreactivity of c-Fos in the spinal cord was compared between young adult (2-3 years in dogs and 6 months in mice) and aged (10-12 years in dogs and 24 months in mice) Beagle dogs and C57BL/6J mice. In addition, changes to neuronal distribution and damage to the spinal cord were also investigated. There were no significant differences in neuronal loss or degeneration of the spinal neurons observed in either the aged dogs or mice. Weak c-Fos immunoreactivity was observed in the spinal neurons of the young adult animals; however, c-Fos immunoreactivity was markedly increased in the nuclei of spinal neurons in the aged dogs and mice, as compared with that of the young adults. In conclusion, c-Fos immunoreactivity was significantly increased without any accompanying neuronal loss in the aged
\end{abstract}

Correspondence to: Professor Moo-Ho Won, Department of Neurobiology, School of Medicine, Kangwon National University, 1 Kangwondaehak, Chuncheon, Gangwon 200-701, Republic of Korea E-mail: mhwon@kangwon.ac.kr

Professor Hyung-Cheul Shin, Department of Physiology, College of Medicine, Hallym University, 1 Hallymdaehak, Chuncheon, Gangwon 200-702, Republic of Korea

E-mail: hcshin@hallym.ac.kr

${ }^{*}$ Contributed equally

Key words: aging, beagle dog, C57BL/6J mouse, spinal gray matter, spinal neurons spinal cord of mice and dogs, as compared with the spinal cords of the young adult animals.

\section{Introduction}

Unexplained pain has been reported as a common complaint of the elderly, and age-related patterns in pain prevalence are complex $(1,2)$. Neuropathic pain, one of the most common types of chronic pain, results from the abnormal processing of sensory input due to damage caused by disorders of the nervous system, such as spinal cord injury, and increases with advancing age $(3,4)$.

c-Fos is a cellular proto-oncogene, belonging to the immediate early gene family. c-Fos has been used as a relative marker of neuronal activity in the brain following numerous types of brain insult (5-7). Furthermore, c-Fos expression in the spinal cord is considered to be a neurotoxic biomarker which has been detected in the dorsal spinal neurons, following nociceptive stimulation (8-12) and repeated swim stress (13).

Previous studies regarding age-related physiological changes, have focussed on spinal cord-specific changes. A loss of myelin and axonal involution have been observed in the aged rat spinal cord (14), and expression levels of substance $P$, a major neurotransmitter of primary afferent nociceptive fibers, and somatostatin have been shown to decrease in the spinal cord of the aged rat $(15,16)$. Little is currently known about the changes in c-Fos expression in the spinal cord during the process of normal aging.

The present study compared the age-related changes in the immunoreactivity of c-Fos in the spinal cords of the young adult and aged Beagle dog and C57BL/6J mouse. Both the Beagle and C57VL/6J mice are considered to be good animal models to study aging (17-21). 


\section{Materials and methods}

Experimental animals. Clinically and neurologically healthy male Beagle dogs and male C57BL/6J mice were used in the present study. Young adult dogs, aged 2-3 years, and aged dogs, aged 10-12 years were used ( $\mathrm{n}=7 /$ group); alongside young adult mice, at 6 months, and aged mice, at 24 months ( $\mathrm{n}=14$ /group). The animals were maintained in conventional housing under adequate temperature $\left(23^{\circ} \mathrm{C}\right)$ and humidity $(60 \%)$ conditions,

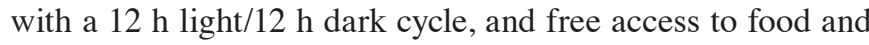
water.

Animal handling and care followed the guidelines of the current international laws and policies [National Institute of Health (NIH) Guide for the Care and Use of Laboratory Animals, NIH Publication no. 85-23, 1985, revised 1996], and the experimental protocol was approved by the Institutional Animal Care and Use Committee of Kangwon National University (approval no. KW-130424-3). All of the experiments were conducted to minimize the number of animals used, and to avoid animal suffering.

Tissue processing for histology. For histochemical analysis, the young adult and aged dogs and mice ( $\mathrm{n}=7$ in each group) were anesthetized with a mixture of Zoletil $50(8 \mathrm{mg} / \mathrm{kg}$; Virbak Korea, Seoul, Korea) Xylazine (2 mg/kg; Bayer Korea, Seoul Korea) and pentobarbital sodium ( $40 \mathrm{mg} / \mathrm{kg}$; JW Phar. Co., Ltd., Seoul, Korea), respectively. Anaesthetization was followed by a transcardial perfusion with $0.1 \mathrm{M}$ phosphate-buffered saline (PBS, pH 7.4; Sigma-Aldrich, St. Louis, MO, USA), followed by $4 \%$ paraformaldehyde (Samchun Chemicals, Pyeongtaek, Korea) in $0.1 \mathrm{M}$ phosphate buffer ( $\mathrm{pH}$ 7.4). The cervical (C6-C8) and lumbar (L5-L6) spinal cord regions were harvested from the animals and postfixed, in the same fixative, for $12 \mathrm{~h}$. The spinal cord tissues were cryoprotected by infiltration with $30 \%$ sucrose (Junsei Chemical Co., Ltd., Tokyo, Japan) overnight. Subsequently, the frozen tissues were serially sectioned at $30 \mu \mathrm{m}$ using a cryostat (Leica, Wetzlar, Germany) and the sections were then placed into six-well plates containing PBS.

Fluoro-Jade B (F-J B) histofluorescence staining. F-J B histofluorescence staining procedures were conducted according to previous methods (22). Briefly, the sections were immersed in a solution of $80 \%$ ethanol containing $1 \%$ sodium hydroxide, followed by immersion in $70 \%$ ethanol. The sections were then transferred into a solution of $0.06 \%$ potassium permanganate, prior to staining with a $0.0004 \%$ F-J B solution (Histochem, Jefferson, AR, USA). The sections were placed on a slide warmer $\left(\sim 50^{\circ} \mathrm{C}\right)$, and examined using an epifluorescent microscope (Carl Zeiss, Oberkochen, Germany) with blue (450-490 nm) excitation light and a barrier filter. This method has been previously reported as being useful in the detection of neuronal degeneration, as degenerating neurons brightly fluoresce in comparison to background fluorescence (23).

Immunohistochemistry for NeuN and c-Fos. Immunohistochemistry for NeuN and c-Fos was performed under the same conditions in both the dogs and the mice of different age groups, in order to determine whether the degree of immunohistochemical staining was accurate. The sections were sequentially treated with $0.3 \% \mathrm{H}_{2} \mathrm{O}_{2}$ and $10 \%$ normal donkey serum (Vector Laboratories, Burlingham, CA, USA). The sections were then incubated with diluted mouse anti-NeuN (1:1,000; Chemicon International, Temecula, CA, USA) and goat anti-c-Fos (1:100; Santa Cruz Biotechnology Inc., Santa Cruz, CA, USA) antibodies. The sections were subsequently exposed to biotinylated horse anti-mouse or rabbit anti-goat antibodies and streptavidin peroxidase complex (1:200; Vector Laboratories Inc., Burlingame, CA, USA). The immunocomplexes were visualized by staining with 3,3'-diaminobenzidine tetrahydrochloride (Sigma-Aldrich, St. Louis, MO, USA) in $0.1 \mathrm{M}$ Tris-HCl buffer ( $\mathrm{pH}$ 7.2) and mounted onto gelatin-coated slides. The sections were mounted in Canada balsam (Kanto Chemical Co., Inc., Portland, OR, USA) following dehydration. In order to establish the specificity of the immunostaining, a negative control test was carried out with pre-immune serum in place of a primary antibody. The negative control test resulted in the absence of immunoreactivity in all structures.

Data analysis. All measurements were performed double blind, in order to ensure objectivity, and the measures of both the control and experimental samples were carried out under the same conditions. NeuN-immunoreactive neurons in the young adult and aged dogs and mice were quantified from 10 sections/each animal, using Optimas 6.5 image analyzing system (CyberMetrics, Scottsdale, AZ, USA) equipped with a computer-based charge coupled device camera. The cell counts were obtained by averaging the counts from the sections taken from each animal. The data rpesented are representative of a percentage of the young adult group.

Ten sections per animal were selected to quantitatively analyze c-Fos immunoreactivity. Digital images of the spinal cord were captured using an Axio M1 light microscope (Carl Zeiss) equipped with a digital camera (Axiocam; Carl Zeiss), connected to a PC monitor. The staining intensity of the c-Fos-immunoreactive structures was evaluated on the basis of a relative optical density (ROD), which was obtained after the transformation of the mean gray level using the following formula: $\mathrm{ROD}=\log (256 /$ mean gray level). The ROD of the complete field was measured, and the brightness and contrast of each image file was calibrated using Adobe Photoshop version 8.0 (Adobe Systems Incorporated, San Jose, CA, USA). The images were then analyzed using the NIH Image 1.59 software (National Institutes of Health, Bethesda, MD, USA). The values of the background staining were obtained and subtracted from the immunoreactive intensities. The data was presented as $\%$, with the young adult group designated as $100 \%$.

Western blot analysis for c-Fos. To confirm the changes in the c-Fos expression levels in the cervical spinal cord region between the young adult and aged mice, the mice spinal cord tissues ( $\mathrm{n}=7$ in each group) were used for western blot analysis. Briefly, the tissues were homogenized in $50 \mathrm{mM}$ PBS (pH 7.4), containing ethylene glycol tetraacetic acid ( $\mathrm{pH} \mathrm{8.0),}$ $0.2 \% \mathrm{NP}-40,10 \mathrm{mM}$ ethylenediaminetetraacetic acid ( $\mathrm{pH} 8.0)$, $15 \mathrm{mM}$ sodium pyrophosphate, $100 \mathrm{mM} \beta$-glycerophosphate, $50 \mathrm{mM}$ sodium fluoride, $150 \mathrm{mM}$ sodium chloride, $2 \mathrm{mM}$ sodium orthovanadate, $1 \mathrm{mM}$ phenylmethanesulfonyl fluoride, and $1 \mathrm{mM}$ dithiothreitol (DTT; Santa Cruz Biotechnology, Inc.). Following centrifugation at $16,000 \mathrm{x}$ g for $20 \mathrm{~min}$, the protein concentration of the supernatants was determined using a Micro 

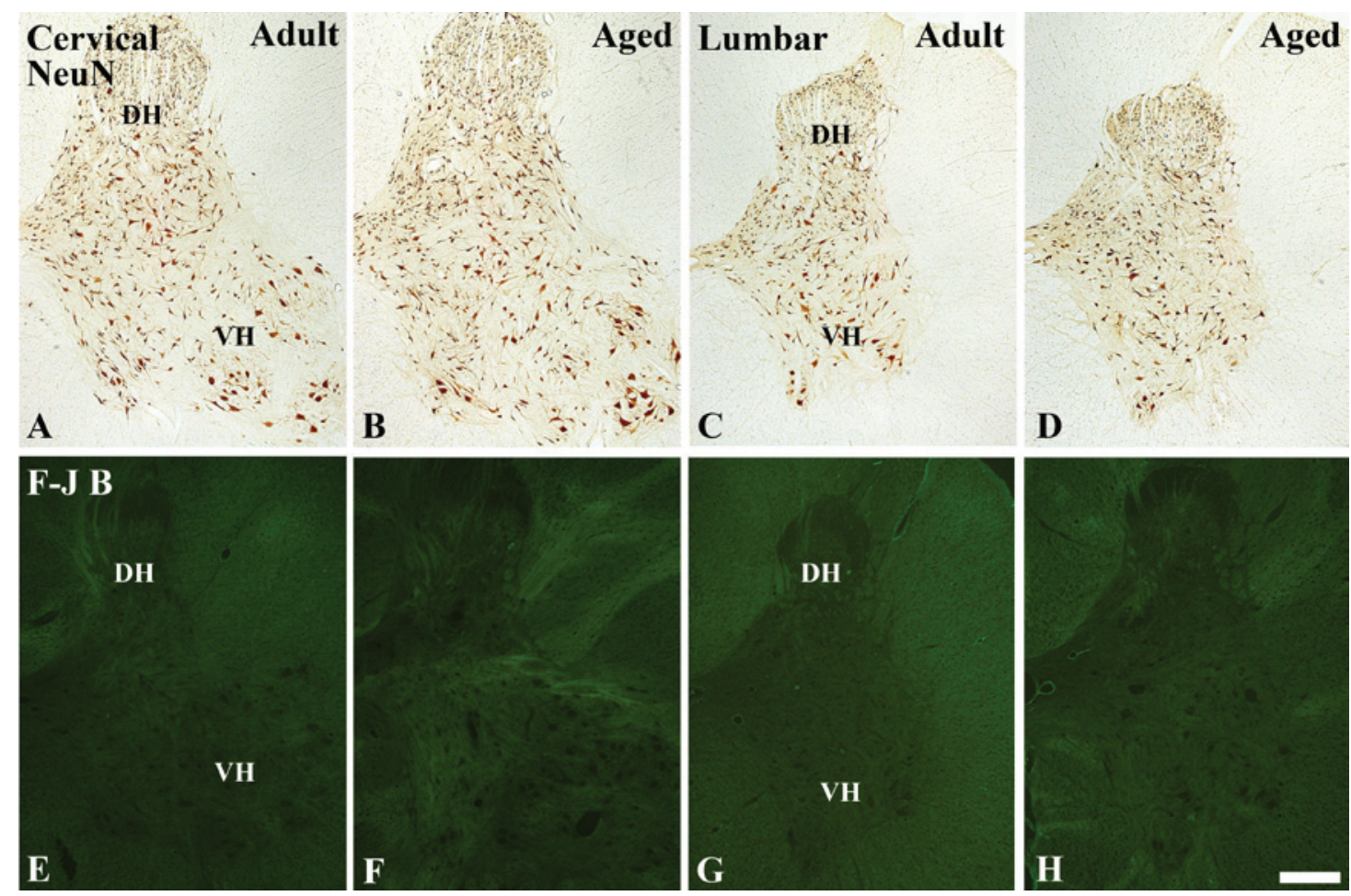

Figure 1. (A-D) Immunohistochemical staining for NeuN immunoreactivity and (E-H) Fluoro-Jade B histofluorescence in the (A, B, E and F) cervical and (C, $\mathrm{D}, \mathrm{G}$ and $\mathrm{H}$ ) lumbar spinal cord regions of the (A, C, E and G) young adult and (B, D, F and H) aged dogs. There were no significant differences in neuronal loss in the young adult or aged dogs. DH, dorsal horn; VH, ventral horn. Magnification, x5; Scale bar $=500 \mu \mathrm{m}$.

Bicinchoninic Acid Protein Assay kit (Pierce Biotechnology, Rockford, IL, USA). Aliquots containing $20 \mu \mathrm{g}$ of total protein were boiled in a loading buffer containing $150 \mathrm{mM}$ Tris (pH 6.8), 3 mM DTT, 6\% SDS, 0.3\% bromophenol blue, and $30 \%$ glycerol. Subsequently, the aliquots were loaded onto a polyacrylamide gel, and separated by electrophoresis, after which the blots were transferred to nitrocellulose membranes (Pall Corporation, Port Washington, NY,USA). The membranes were incubated with 5\% non-fat dry milk in PBS containing $0.1 \%$ Tween-20, followed by an incubation with the primary antibody for $2 \mathrm{~h}$. The membranes were then incubated with peroxidase-conjugated donkey anti-goat immunoglobulin $\mathrm{G}$ (Sigma-Aldrich). An Enhanced Chemiluminescence kit (Pierce Biotechnology) was used to visualize the blots. Western blot analysis was repeated three times. Following exposure of the membranes, the blots were scanned and densitometric analysis for the quantification of the bands was performed using Scion Image software (Scion Corporation, Frederick, MD, USA), which was used to determine the ROD. A ratio of the ROD was calibrated as a percentage, with the young adult group designated as $100 \%$.

Statistical analysis. The difference of the mean ROD between the groups was statistically analyzed using a Student t-test. A $\mathrm{P}<0.05$ was considered to indicate a statistically significant difference.

\section{Results}

NeuN-immunoreactive neurons. NeuN-immunoreactive neurons were shown to be distributed throughout the grey matter of the cervical and lumbar spinal cord regions in the young adult and aged dogs (Fig. 1A-1D). There were no significant differences in the number of NeuN-immunoreactive neurons between the young adult and aged dogs, however the number of NeuN-immunoreactive neurons was shown to be slightly decreased in the cervical and lumbar regions of the aged spinal cord, as compared with the number in the young adult spinal cord (data not shown).

Similarly to the dogs, the number of NeuN-immunoreactive neurons in the cervical and lumbar spinal cord regions was not significantly different between the young adult and aged mice (data not shown).

F-J B positive cells. To examine the extent of neuronal degeneration of the spinal cord, F-J B staining was performed in the young adult and aged dogs and mice. F-J B positive cells were not observed in the cervical and lumbar spinal cord regions of the young adult and aged dogs (Fig. 1E-H) or mice (data not shown).

c-Fos immunoreactivity. In the young adult dogs, moderate c-Fos immunoreactivity was observed in the neurons in the whole grey matter of the cervical and lumbar spinal cord regions (Fig. 2A, 2b, 2C and 2f). The c-Fos immunoreactivity was generally found to be contained within the nuclei of the spinal neurons (Fig. $2 \mathrm{~b}$ and $2 \mathrm{f}$ ). In the aged dogs, the c-Fos expression pattern was similar to that in the young adult dogs (Fig. 2B, 2c, 2d, 2D, 2g, and 2h); however, the c-Fos immunoreactivity in the nuclei of the neurons in the aged dogs was significantly higher as compared with that in the young adult dogs (Fig. 2c, 2d, 2g, 2h and 2E) ( $\mathrm{P}<0.05)$.

c-Fos immunoreactivity was also observed in the nuclei of the spinal neurons of the cervical and lumbar spinal cord 

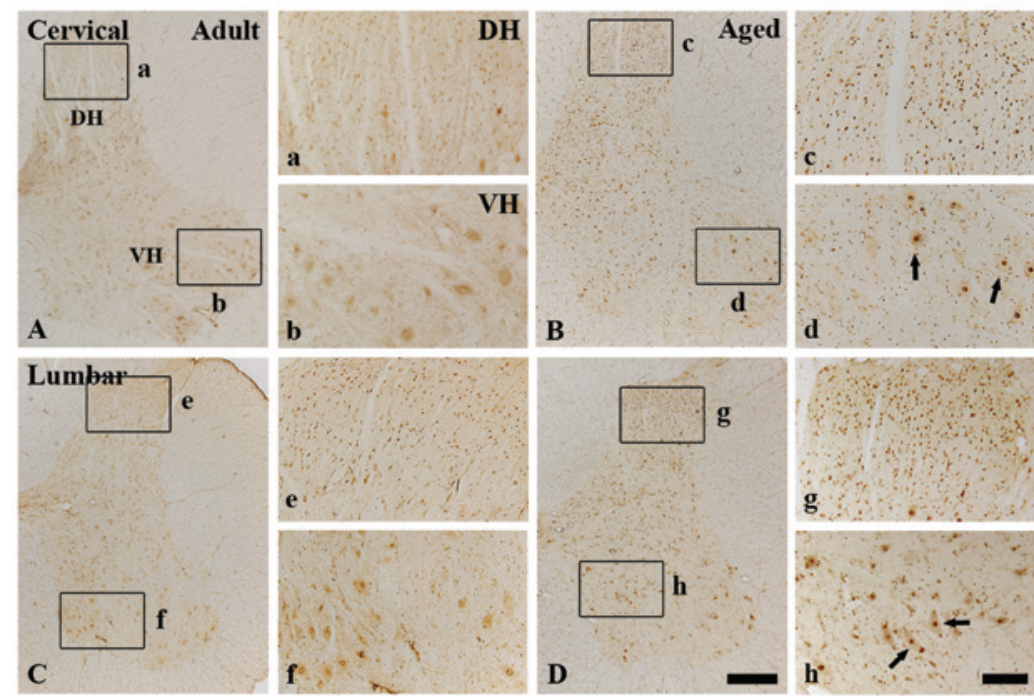

E

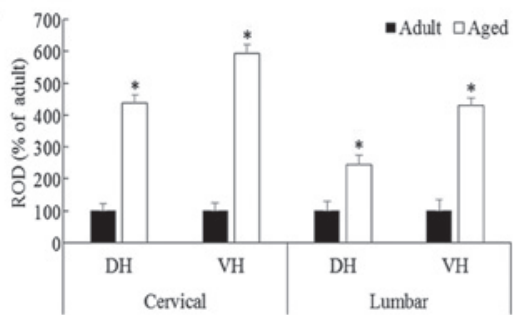

Figure 2. (A and B) c-Fos immunohistochemistry in the cervical and (C and D) lumbar spinal cord regions of (A and C) young adult and (B and D) aged dogs. c-Fos immunoreactivity (arrows in "c, d, g and h") was increased in the aged group. Strong immunoreactivity was observed in the nuclei of the spinal neurons. Scale bars $=(A-D) 500 \mu \mathrm{m},(\mathrm{a}-\mathrm{h}) 50 \mu \mathrm{m}$. (E) Relative optical density as a percentage of c-Fos-immunoreactive structures in the young adult and aged spinal cord ( $\mathrm{n}=7$ per group; $\mathrm{P}<0.05$ versus the adult group). The bars indicate the means \pm standard error of the mean. $\mathrm{DH}$, dorsal horn; $\mathrm{VH}$, ventral horn; ROD, relative optical density.

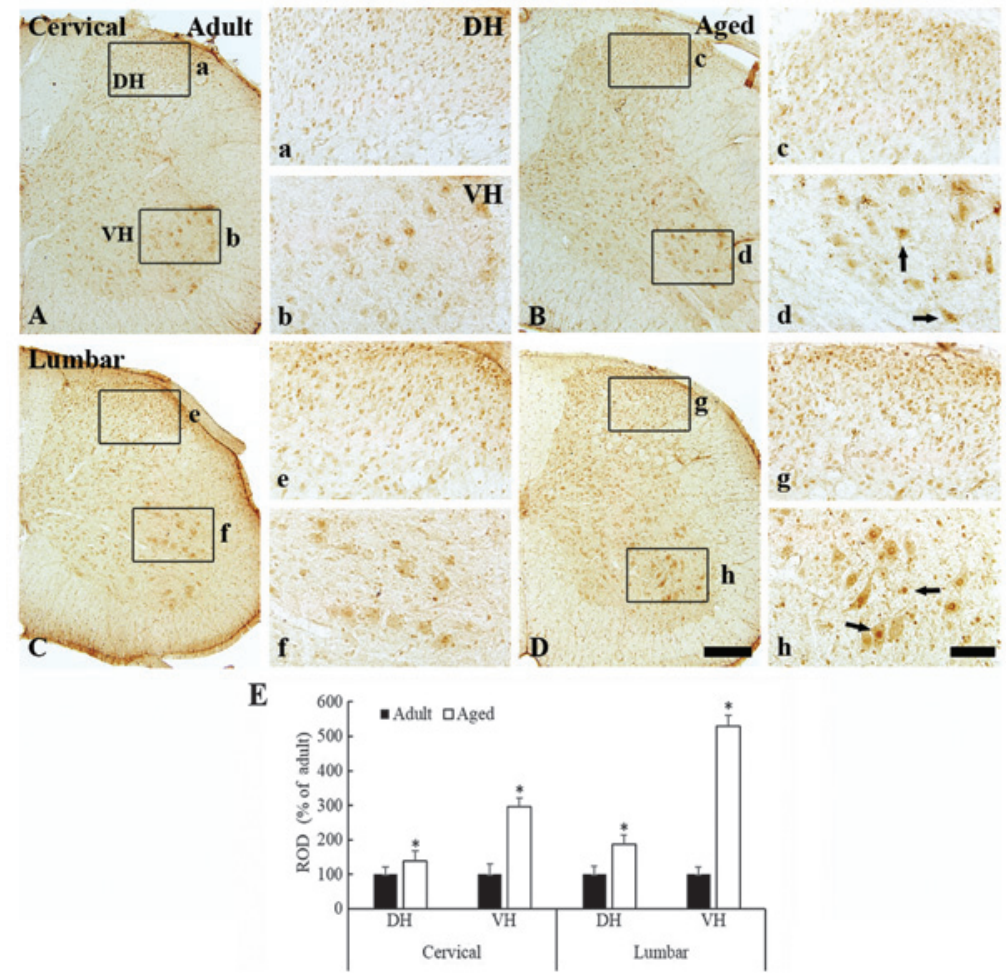

Figure 3. (A and B) c-Fos immunohistochemistry in the cervical and (C and D) lumbar spinal cord regions of (A and C) young adult and (B and D) agedmice. c-Fos immunoreactivity (arrows in "c, d, g and h") was increased in the aged group. Strong immunoreactivity was observed in the nuclei of the spinal neurons. Scale bars $=$ (A-D) $50 \mu \mathrm{m}$, (a-h) $200 \mu \mathrm{m}$. (E) Relative optical density as a percentage of c-Fos-immunoreactive structures in the young adult and aged spinal cord ( $\mathrm{n}=7$ per group; ${ }^{\mathrm{P}}<0.05$ versus the adult group). The bars indicate the means \pm standard error of the mean. $\mathrm{DH}$, dorsal horn; VH, ventral horn; ROD, relative optical density. 

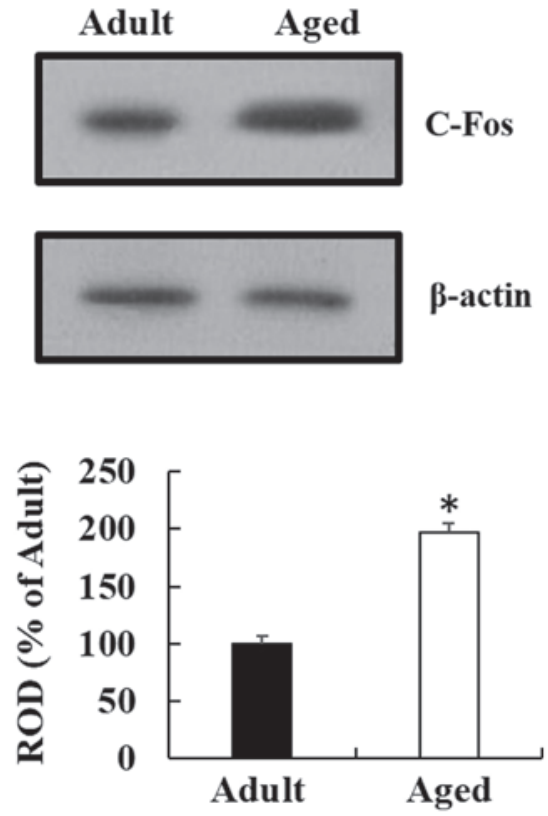

Figure 4. Western blot analysis of c-Fos protein expression levels in the cervical spinal cord region derived from young adult and aged mice. Relative optical density of immunoblot bands is demonstrated as a percentage of the values ( $\mathrm{n}=7$ per group; ${ }^{\mathrm{P}}<0.05$ versus the adult group). The data are presented as the means \pm standard error of the mean. ROD, relative optical density.

regions in both the young adult and aged mice (Fig. 3A-3D). Similarly to the aged dogs, c-Fos immunoreactivity was significantly increased in the aged mice as compared with the young adult mice. This increase in immunoreactivity was observed in both the dorsal (Fig. 3c and 3g) and the ventral horn (Fig. 3d and 3h) of the cervical and lumbar spinal cord regions (Fig. 3E) $(\mathrm{P}<0.05)$.

c-Fos protein levels. Western blot analysis indicated that the pattern of change in the c-Fos protein expression levels, in the cervical spinal cord region of the aged mice, was similar to that observed by immunohistochemical analysis. c-Fos protein expression in the cervical spinal cord region of the aged mice was significantly increased, as compared with the expression in the young adult mice (Fig. 4) $(\mathrm{P}<0.05)$.

\section{Discussion}

Age-related pain is associated with a poor quality of life, physical disability and an increased risk of mortality $(24,25)$. In the present study, c-Fos immunoreactivity in the spinal cord was compared between young adult and aged dogs and mice. Changes to the distribution and damage/death of spinal neurons were also investigated in both the young adult and aged spinal cords.

There was no significant difference observed in the number of NeuN-immunoreactive neurons between the spinal cords of the young adult and aged dogs and mice. In addition, F-J B positive degenerating neurons were not detected in the spinal cord of either the young adult or aged animals. This finding is consistent with findings from our previous studies, which reported that no distinct neuronal loss was observed in the aged spinal cord of German shepherd dogs $(26,27)$.
The present study found that weak c-Fos immunoreactivity was observed in the dorsal and ventral spinal neurons of the young adult dogs and mice, whereas the c-Fos immunoreactivity was significantly increased in the aged dogs and mice as compared with the young adult groups. The present findings support previous research that showed that low levels of c-Fos immunoreactivity was observed in young adult animals (28-30). However, other studies have previously reported that the basal expression of c-Fos in the dorsal horn was decreased in the aged rat spinal cord (31), which is not consistent with the findings of the present study. This discrepancy may result from differences in experimental methods.

It has previously been reported that c-Fos and astrocytes become activated by a combined stress of non-thermal irradiation and the toxic effects of picrotoxin in the rat brain (32). Activated astrocytes are associated with several neuropathic and cancer pain states, through the release of neurotoxic substances including pro-inflammatory cytokines (33-35). In addition, it has previously been reported that pro-inflammatory cytokines, including interferon- $\gamma$, and interleukins (IL)-1 $\beta$ and -2, were markedly increased, without any significant neuronal loss, in the spinal cord of the aged $\operatorname{dog}(26,36)$. It has also been reported that increasing age can enhance the expression levels of tumor necrosis factor- $\alpha$, the IL- 6 family of cytokines, chemokine receptor- 2 and pro-inflammatory chemokines following ischemic stroke in the aged rat (37). Therefore, based on these data and the results of the present study, c-Fos expression in the spinal cord may be triggered by different stress factors, including chronic inflammatory activity.

In conclusion, the present study observed that c-Fos immunoreactivity in the aged dog and mouse spinal cords was markedly increased, as compared with young adult animals. This finding implicates that the increase in the expression of c-Fos in the aged dog spinal cord may be associated with aging-related changes in the aged spinal cord.

\section{Acknowledgements}

The authors would like to thank Mr. Seung Uk Lee for his technical help in this study. This research was supported by the National Research Foundation of Korea funded by the Ministry of Education, Science and Technology (2010-0010580) and by the ICT R\&D program of MSIP/IITP (10033634).

\section{References}

1. Gibson SJ and Helme RD: Age-related differences in pain perception and report. Clin Geriatr Med 17: 433-456, 2001.

2. Helme RD and Gibson SJ: The epidemiology of pain in elderly people. Clin Geriatr Med 17: 417-431, 2001.

3. Werhagen L, Budh CN, Hultling C and Molander C: Neuropathic pain after traumatic spinal cord injury - relations to gender, spinal level, completeness, and age at the time of injury. Spinal Cord 42: 665-673, 2004

4. Schmader KE: Epidemiology and impact on quality of life of postherpetic neuralgia and painful diabetic neuropathy. Clin J Pain 18: 350-354, 2002.

5. Coggeshall RE: Fos, nociception and the dorsal horn. Prog Neurobiol 77: 299-352, 2005.

6. Munglani R, Hudspith MJ, Fleming B, et al: Effect of pre-emptive NMDA antagonist treatment on long-term Fos expression and hyperalgesia in a model of chronic neuropathic pain. Brain Res 822: 210-219, 1999. 
7. Lu Y and Westlund KN: Effects of baclofen on colon inflammation-induced Fos, CGRP and SP expression in spinal cord and brainstem. Brain Res 889: 118-130, 2001.

8. Abbadie C, Besson JM and Calvino B: c-Fos expression in the spinal cord and pain-related symptoms induced by chronic arthritis in the rat are prevented by pretreatment with Freund adjuvant. J Neurosci 14: 5865-5871, 1994.

9. Hwang HJ, Lee HJ, Kim CJ, Shim I and Hahm DH: Inhibitory effect of amygdalin on lipopolysaccharide-inducible TNF-alpha and IL-1beta mRNA expression and carrageenan-induced rat arthritis. J Microbiol Biotechnol 18: 1641-1647, 2008.

10. Liu CR, Duan QZ, Wang W, et al: Effects of intrathecal isoflurane administration on nociception and Fos expression in the rat spinal cord. Eur J Anaesthesiol 28: 112-119, 2011.

11. Wu J, Hu Q, Huang D, Chen X and Chen J: Effect of electrical stimulation of sciatic nerve on synaptic plasticity of spinal dorsal horn and spinal c-fos expression in neonatal, juvenile and adult rats. Brain Res 1448: 11-19, 2012.

12. Zeng X, Huang H and Hong Y: Effects of intrathecal BAM22 on noxious stimulus-evoked c-fos expression in the rat spinal dorsal horn. Brain Res 1028: 170-179, 2004.

13. Quintero L, Cuesta MC, Silva JA, et al: Repeated swim stress increases pain-induced expression of c-Fos in the rat lumbar cord. Brain Res 965: 259-268, 2003.

14. Terao S, Sobue G, Hashizume Y, Shimada N and Mitsuma T: Age-related changes of the myelinated fibers in the human corticospinal tract: a quantitative analysis. Acta Neuropathol 88: 137-142, 1994

15. Hukkanen M, Platts LA, Corbett SA, Santavirta S, Polak JM and Konttinen YT: Reciprocal age-related changes in GAP-43/B-50, substance $\mathrm{P}$ and calcitonin gene-related peptide (CGRP) expression in rat primary sensory neurones and their terminals in the dorsal horn of the spinal cord and subintima of the knee synovium. Neurosci Res 42: 251-260, 2002.

16. Ranson RN, Priestley DJ, Santer RM and Watson AH: Changes in the substance P-containing innervation of the lumbosacral spinal cord in male Wistar rats as a consequence of ageing. Brain Res 1036: 139-144, 2005

17. Head E, Nukala VN, Fenoglio KA, Muggenburg BA, Cotman CW and Sullivan PG: Effects of age, dietary, and behavioral enrichment on brain mitochondria in a canine model of human aging. Exp Neurol 220: 171-176, 2009.

18. González-Martínez Á, Rosado B, Pesini P, et al: Plasma $\beta$-amyloid peptides in canine aging and cognitive dysfunction as a model of Alzheimer's disease. Exp Gerontol 46: 590-596, 2011

19. Barańczyk-Kuźma A, Usarek E, Kuźma-Kozakiewcz M, et al: Age-related changes in tau expression in transgenic mouse model of amyotrophic lateral sclerosis. Neurochem Res 32: 415-421, 2007.

20. Garbuzova-Davis S, Haller E, Saporta S, Kolomey I, Nicosia SV and Sanberg PR: Ultrastructure of blood-brain barrier and blood-spinal cord barrier in SOD1 mice modeling ALS. Brain Res 1157: 126-137, 2007.

21. Coksaygan T, Magnus T, Cai J, et al: Neurogenesis in Talpha-1 tubulin transgenic mice during development and after injury. Exp Neurol 197: 475-485, 2006.
22. Candelario-Jalil E, Alvarez D, Merino N and León OS: Delayed treatment with nimesulide reduces measures of oxidative stress following global ischemic brain injury in gerbils. Neurosci Res 47: 245-253, 2003

23. Schmued LC and Hopkins KJ: Fluoro-Jade B: a high affinity fluorescent marker for the localization of neuronal degeneration. Brain Res 874: 123-130, 2000.

24. Kendig H, Browning CJ and Young AE: Impacts of illness and disability on the well-being of older people. Disabil Rehabil 22: 15-22, 2000.

25. Thomas E, Peat G, Harris L, Wilkie R and Croft PR: The prevalence of pain and pain interference in a general population of older adults: cross-sectional findings from the North Staffordshire Osteoarthritis Project (NorStOP). Pain 110: 361-368, 2004.

26. Chung JY, Choi JH, Lee CH, et al: Comparison of ionized calcium-binding adapter molecule 1-immunoreactive microglia in the spinal cord between young adult and aged dogs. Neurochem Res 35: 620-627, 2010

27. Ahn JH, Choi JH, Kim JS, et al: Comparison of immunoreactivities in 4-HNE and superoxide dismutases in the cervical and the lumbar spinal cord between adult and aged dogs. Exp Gerontol 46: 703-708, 2011.

28. Lawrence J, Stroman PW, Bascaramurty S, Jordan LM and Malisza KL: Correlation of functional activation in the rat spinal cord with neuronal activation detected by immunohistochemistry. Neuroimage 22: 1802-1807, 2004

29. Bullitt E: Expression of c-fos-like protein as a marker for neuronal activity following noxious stimulation in the rat. J Comp Neurol 296: 517-530, 1990.

30. Wu YP and Ling EA: Expression of Fos in the spinal motoneurons labelled by horseradish peroxidase following middle cerebral artery occlusion in rat. Brain Res Bull 45: 571-576, 1998.

31. Kim JM, Lee KW, Chung YH, Shin CM, Baik SH and Cha CI: c-Fos basal immunoreactivity decreases in rat spinal cord during normal ageing. Neuroreport 10: 585-588, 1999.

32. Carballo-Quintás M, Martínez-Silva I, Cadarso-Suárez C, et al: A study of neurotoxic biomarkers, c-fos and GFAP after acute exposure to $\mathrm{GSM}$ radiation at $900 \mathrm{MHz}$ in the picrotoxin model of rat brains. Neurotoxicology 32: 478-494, 2011.

33. Sun YN, Luo JY, Rao ZR, Lan L and Duan L: GFAP and Fos immunoreactivity in lumbo-sacral spinal cord and medulla oblongata after chronic colonic inflammation in rats. World $\mathrm{J}$ Gastroenterol 11: 4827-4832,2005.

34. Hald A, Nedergaard S, Hansen RR, Ding M and Heegaard AM: Differential activation of spinal cord glial cells in murine models of neuropathic and cancer pain. Eur J Pain 13: 138-145, 2009.

35. Watkins LR, Milligan ED and Maier SF: Glial activation: a driving force for pathological pain. Trends Neurosci 24: 450-455, 2001.

36. Lee DH, Ahn JH, Park JH, et al: Comparison of expression of inflammatory cytokines in the spinal cord between young adult and aged beagle dogs. Cell Mol Neurobiol 33: 615-624, 2013.

37. Dinapoli VA, Benkovic SA, Li X, et al: Age exaggerates proinflammatory cytokine signaling and truncates signal transducers and activators of transcription 3 signaling following ischemic stroke in the rat. Neuroscience 170: 633-644, 2010. 